\title{
The power of a positive approach to realizing change in an animal facility
}

To the Editor - A rodent facility must meet the needs of its animal population at the most fundamental levels of food, water, and housing. Because related husbandry tasks are performed daily and the animals are thriving, complacency can set in with current practices. Nevertheless, detailed examination of a process can often yield opportunity for improvement. Our team routinely analyzes current practices and seeks out innovative and creative ways of improving efficiency while maintaining or enhancing animal welfare standards for our rodents.

One process that we recently examined was the application of pellets into feed hoppers, an essential activity that is performed on all cages. We determined that there was a pervasive technician practice of overfilling feed hoppers in our facility.

This practice can have negative consequences. For instance, placing excess feed in the hopper results in wasted feed at the time of discard, and thus, a loss of money. Additionally, excess pellets typically spread over part of the surface of the wire bar lid; this can potentially obstruct proper airflow within ventilated cages and result in elevated intracage ammonia levels, which in turn could alter animal health and wellbeing. Lastly, this practice can be a hindrance to researchers, veterinarians, and health technicians, as excess pellets on a wire bar lid often make replacement of a bonnet onto a cage cumbersome.

To solve this problem, it was necessary to understand the cause. Following an informal investigation, we determined that it was multifactorial. The tools that our technicians were using promoted overfilling, as we had historically provided oversized scoops. Also, we recognized that technicians, like most people, prefer to get the job done quickly. For this task to be most efficient, they found that overfilling 'today' makes topping off 'tomorrow' quicker: they could later simply tip a cage forward, allowing the excess feed to roll back into the hopper. Thus, overfilling reduced the frequency and effort needed to refill the food hoppers by eliminating the step of physically scooping new food into the hopper.
We then analyzed our feed application process. At the start of a 4-week period, cage bottoms and hoppers are changed, and hoppers are filled on Day 1. Cage bottoms are changed and a scheduled feed top-off event is performed on Day 14. Both cage bottoms and hoppers are renewed on Day 28. However, approximately $50 \%$ of cages require an additional top-off in between scheduled cage changes; that is, between days 1 and 14 and then again between days 14 and 28. This results in an average of 2 top-off events per cage during each 4 week cycle.

At the conclusion of the 4-week cycle, all feed is discarded. The loss of feed from the $50 \%$ of cages that are provided excess feed in the second half of the cycle, when compared to cages topped off correctly, is approximately $3.5 \mathrm{oz}$. The $3.5 \mathrm{oz}$ of excess feed that is discarded results in a loss of $\$ .16$ per cage. In our facility, we have approximately 20,000 cages; thus, approximately 10,000 result in a loss of excess feed per cycle. If we prevented excess top-offs on all cages, we could potentially save $\$ 1600$ per month.

But to achieve our goal, we had to change a longstanding practice and overcome a natural resistance to change embedded behaviors that were comfortable and familiar to our technicians. We elected to approach this challenge with the use of positive reinforcement.

We began with a broad discussion of information in an assembly setting. We initially provided personnel with a description of the problem, our analysis of the causes, and benefits of change. We then provided greater detail and interactive discussion to encourage technician participation in smaller team settings within the vivarium. On a weekly basis, we subsequently walked through the facility and met one-on-one with each individual, cage-side during change-out, to provide positive feedback on their progress. Thus, each individual received consistent and repeated recognition and affirmations to foster the new behaviors. Furthermore, we provided new tools-smaller scoops-and standardized the amount of feed to be added.
Lastly, to facilitate further engagement in the process, we created a friendly competition between teams working in different areas of the vivarium and offered a "prize" luncheon to the team that successfully adopted the desired behaviors within our stated timeframe of 12 weeks. Ultimately, all teams succeeded and received the luncheon to celebrate. The competition improved our facility practices while solidifying our teams and supporting morale.

To summarize, through routine analysis of our daily practices we identified a single, simple practice that had a significant cost. Following analysis of the causes and motivations behind the practice, we used a simple, stepwise process to change it. Over a 12-week period, we were able to modify the behavior and culture of our personnel. We have discontinued the practice of overfilling feed hoppers in our entire facility of approximately 20,000 cages, resulting in significant financial benefit to the institution. Moreover, we removed the potential for airflow obstruction within the cage, and improved the day-to-day working experience for our research and health care personnel. We accomplished this by using the thoughtful application of positive reinforcement techniques with our personnel which resulted in a smooth, painless, and lasting change in practice and demonstrated that the culture of a team can change while realizing necessary improvements. In the process, we strengthened relationships between management and technicians. Moreover, we have established positive expectations for future change, such that there should be increased receptivity and openness to the prospect of modifications to other longstanding practices.

\section{Cynthia R. Lockworth ${ }^{\star}$, Tom Rodriguez Jr, Matthew S. Schmit and Lorraine R. Hill University of Texas MD Anderson Cancer Center, Houston, TX, USA. \\ *e-mail: crlockworth@mdanderson.org}

Published online: 26 February 2018 https://doi.org/10.1038/s41684-018-0001-3 